\title{
Communicating Phylogeny: Evolutionary Tree Diagrams in Museums
}

\author{
Teresa MacDonald • E. O. Wiley
}

Published online: 2 March 2012

(C) Springer Science+Business Media, LLC 2012

\begin{abstract}
Tree of life diagrams are graphic representations of phylogeny - the evolutionary history and relationships of lineages - and as such these graphics have the potential to convey key evolutionary ideas and principles to a variety of audiences. Museums play a significant role in teaching about evolution to the public, and tree graphics form a common element in many exhibits even though little is known about their impact on visitor understanding. How phylogenies are depicted and used in informal science settings impacts their accessibility and effectiveness in communicating about evolution to visitors. In this paper, we summarize the analysis of 185 tree of life graphics collected from museum exhibits at 52 institutions and highlight some potential implications of how trees are presented that may support or hinder visitors' understanding about evolution. While further work is needed, existing learning research suggests that common elements among the diversity of museum trees such as the inclusion of anagenesis and absence of time and shared characters might represent potential barriers to visitor understanding.
\end{abstract}

Keywords Evolutionary trees · Trees of life · Phylogeny · Museums

T. MacDonald $(\bowtie)$

University of Kansas Natural History Museum,

University of Kansas,

1345 Jayhawk Blvd, Dyche Hall,

Lawrence, KS 66045-7593, USA

e-mail: tmacd@ku.edu

E. O. Wiley

Ecology \& Evolutionary Biology and Biodiversity Institute,

University of Kansas,

Lawrence, KS 66045-7593, USA
The idea of a "tree of life" represents a core concept of evolutionary science - phylogeny-and is depicted graphically using an almost bewildering array of formats and terminology, in which even a particular geometry used can have multiple names associated with it. Evolutionary trees of life are branching diagrams that depict hypothesized relationships - the historical pattern of divergence and descent between taxa-as a series of branches that merge at internal branches representing common ancestry, which in turn are connected with more distant relatives. As visual representations of the history of lineages or phylogeny, trees reflect the core concept of common ancestry. The importance of phylogeny in supporting understanding of evolution is highlighted in key education documents (American Association for the Advancement of Science 2001; Baum et al. 2005; National Research Council 1996). Tree diagrams, as a graphical representation of this principle, have the potential to play a valuable role in conveying evolutionary ideas.

How people interpret and understand evolutionary trees is a complex interaction between their prior knowledge and understanding of underlying evolutionary ideas such as similarity, ancestry, and relatedness, and their ability to read the relationships depicted in a schematic tree diagram. Given the diversity of tree depictions, one might ask what people understand from these different graphic representations. Many of the common misconceptions about reading and interpreting tree diagrams are well established (Gregory 2008; Meir et al. 2007), and work has been and continues to be done on the use of trees with students in structured learning environments (Baum et al. 2005; Halverson 2010; Novick and Catley 2007; Novick et al. 2010a, b). However, there is a gap in our knowledge about how trees are used and understood outside of a formal instructional framework. An understanding of how, and in what form, tree graphics are 
used in informal settings is an important part of supporting the development of evolutionary thinking in museum visitors.

Museums are an important part of how the public accesses science information, including evolution, and in teaching about these ideas to their visitors (Diamond and Evans 2007; National Science Board 2008). In fact, a recent study found that even a single visit to an evolution exhibit can influence children's thinking about evolutionary concepts (Diamond et al. 2012). Evaluation studies with natural history museum visitors shows that they are interested in the tree of life, but struggle with interpreting the content and relationships represented in trees (Giusti and Scott 2006; Spiegel et al. 2006). While few museums use phylogeny as an organizing principle in their galleries, evolutionary diagrams form a major graphic element in many museums and other informal science settings (Diamond and Scotchmoor 2006; MacDonald 2010). Tree diagrams as a way of representing relatedness is a pervasive element in exhibits that extends beyond science institutions; for example, even the Creation Museum in Kentucky contrasts evolutionary trees with a series of trees depicting separately created kinds, including a solitary and independent line for humans.

In natural history museums, visitors can see a wide range of historical depictions of the tree of life, depending on when an exhibit was developed and the research emphasis of the scientific curators. The graphic representation of the tree in each new exhibit usually reflects the current usage or discipline preferences, but since older depictions often are kept on display, a range of different presentations of tree diagrams is depicted even within a single institution (Diamond and Scotchmoor 2006; MacDonald 2010). Some galleries intentionally use more than one depiction of the tree of life to emphasize to visitors the validity of alternative approaches or different elements (Diamond 2005), but often it simply reflects the reality of long-lived exhibits in museum settings.

As Diamond and Scotchmoor (2006) emphasized in their review of evolution exhibits in museums, the way phylogenies are used determines their effectiveness in reinforcing fundamental concepts about evolution, and consideration of the conceptual and developmental issues of how people understand evolution can make such exhibits accessible to more audiences. We strive for scientific accuracy in our exhibits but also need to recognize and accommodate the needs and knowledge of museum visitors - a carefully created and scientifically accurate diagram for a research journal is likely to be inaccessible to many visitors and even other scientists.

Given the diversity of tree depictions, and the bearing this may have on understanding evolution, one might ask what forms of tree of life diagrams museum visitors might encounter, how and what information is presented, and what impact these different representations might have on visitor understanding. With this in mind, a team of cognitive and learning scientists and museum educators initiated the $\mathrm{Na}$ tional Science Foundation-funded Understanding the Tree of Life project (grant no. 0715287) to conduct a series of pilot studies on how trees are understood and to explore evolutionary tree graphics used in informal science settings.

A summary of the descriptive study of museum trees is presented here, and the findings are discussed within the broader context of current learning research literature about how trees are interpreted and understood. In particular, our work builds on the 2008 analysis of evolutionary diagrams in school textbooks by Catley and Novick, which found many graphics to be confusing and likely to reinforce misconceptions about evolution. This study adopts several elements from the classification scheme they developed and extends the discussion to informal learning settings. A collection of museum trees used in this study and summaries of the project's pilot studies can be found at Understanding the Tree of Life website (http://evolution.berkeley.edu/UToL/index.html).

\section{Tree Collection and Analysis}

Images of 185 evolutionary trees used in exhibits were collected from 52 informal science institutions along with metadata such as information about when each one was developed, its source, etc. between May 2008 and February 2009. Source institutions included natural history museums, science centers, zoos, and aquariums from six countries, but primarily in the United States. Details of these institutions and the number of trees shared for this study are listed in Appendix B in a previous paper (see details below). Only one of the trees was developed prior to 1970 . The majority of the remaining trees were developed after 2000 during exhibit renovations; therefore, the sample is weighted towards trees from 1990 and later. Figure 1 shows the breakdown of trees collected by decade and by tree type (see later "Tree Type" section). It is important to note that many institutions have trees that span several

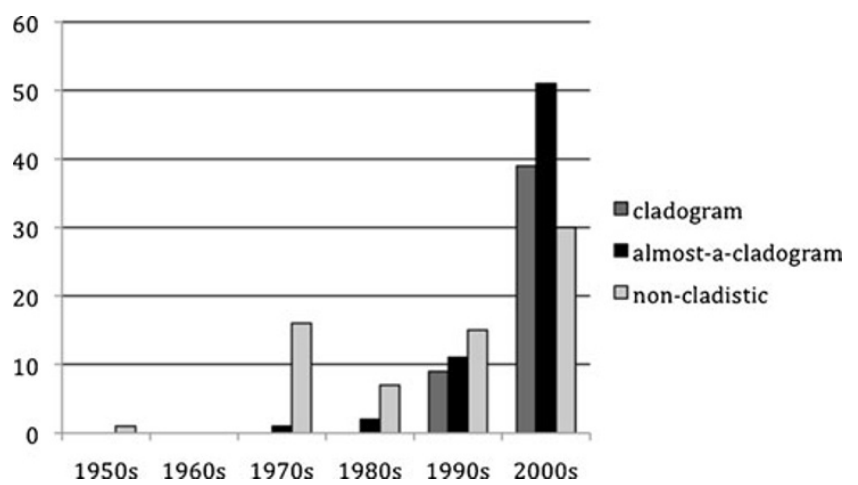

Fig. 1 Distribution of study trees by decade and type 
decades in their exhibitions; therefore, visitors are often exposed to a range of graphics during their visit.

Each graphic was coded according to its features in four categories: tree type, topology (e.g., orientation, geometry), content, and mode of presentation (e.g., graphic panel, kiosk). Only a subset is presented here-detailed descriptions of all categories, coding schemes and results were presented as a paper at the National Association for Research in Science Teaching annual conference, March 2010 in Pittsburgh, PA, USA (publically available at http://evolution.berkeley.edu/ UToL/macdonald_NARST2010.pdf). The categories selected reflect an attempt to examine the accuracy of the evolutionary science content and their potential educational efficacy in the context of existing research on understanding and teaching about phylogeny and the tree of life.

Data was entered into SPSS (SPSS Inc., Version 17.0 for Mac OS X) and appropriate statistical tests were run-frequency distributions for summaries of tree topology and content, chi-square for testing associations between variables, and Fisher's exact test for those cases with a small sample size.

\section{Tree Type}

The categories and coding criteria for tree type were developed using a preliminary assessment of sample trees, a review of existing classification schemes used for biology textbook trees (see Catley and Novick 2008; Donovan and Wilcox 2004), and in discussion with a systematist and others. Diagrams were coded into three categories (outlined below) cladogram, almost-a-cladogram, and non-cladistic/ other evolutionary trees. These categories were chosen not only to allow for comparison to prior work on textbook trees but also to reflect the complexity and diversity seen in museum diagrams. Statistical tests were used to assess inter-coder reliability for tree type and refine category definitions (kappa $=0.929, p<0.001$; a score of 1.0 indicates $100 \%$ agreement).

The categories are based on the overall representations used in the trees, and not on any descriptors that might be associated with the tree- - e.g., whether or not it was labeled as a cladogram. Few diagrams can be tied to a particular research paper, and the data sets, assumptions, and methodologies used to build the trees are not available or are unknown. Without this information, it is not always possible to determine if the groups represented are monophyletic (groups that contain the most recent common ancestor and all descendants) as opposed to paraphyletic (groups that do not include all descendants from an ancestor) or polyphyletic (groups that do not include their common ancestor) (Wiley 1979, 1981) - and so unless it was obvious that they are not, the assumption is made that the groups are monophyletic. The significance of this distinction is discussed later.
The three tree type categories and the criteria used are as follows (Fig. 2):

Cladogram. Branching diagrams that depict common ancestry and the pattern of relationships between taxa and only include monophyletic groups and polytomies (unresolved branches). Criteria for inclusion as a valid cladogram follow those used by Catley and Novick (2008) such as terminal taxa end points being at the same level and not including ancestor-descendant relationships. However, unlike their scheme, trees that have labels on branches or nodes other than characters or to define branching events were included, since in many cases these labels refer to classification categories that also reflect shared characteristics (e.g., amniotes).

Almost-a-cladogram. Diagrams that depict patterns of relationship through branching sequence, as described above, but have some diagrammatic variable that precludes it from being considered a valid cladogram as defined by Catley and Novick (2008). This category includes trees with different terminal end points, varying branch thickness, and side branches.

Non-cladistic/other evolutionary trees. Diagrams that depict evolutionary relationships but that do not qualify as a cladogram. This category includes trees without taxa, those with amorphous or indistinct branching patterns, and graphics that:

- Depict ancestor-descendant relationships-anagenesis: (1) there is a specified ancestral species at a node; this does not include generic references to an unknown hypothetical ancestor such as "early primate ancestor", and (2) there are one or more taxa in a sequence along or within a branch. It is possible that these may be intended to represent morphotypes-hypothetical generalized forms having all the shared characters of a group; however, unless specified as such, the assumption is that it violates cladistic principles by including ancestor-descendant statements as defined by Catley and Novick.

- Portray higher-level taxonomic groups (e.g., order or family) as ancestors to other groups, or refer to one group as "coming from," "leading to," or "giving rise" to other taxa.

Descriptions, criteria, and coding used for the topological/ diagrammatic elements, content, presentation, and explanatory items are summarized in Table 1.

\section{Results}

This study found a wide diversity of evolutionary trees used in museum exhibits, often within the same 
Fig. 2 Examples of museum tree types: a cladogram, b almost-a-cladogram, and $\mathbf{c}$ noncladistic/other evolutionary tree

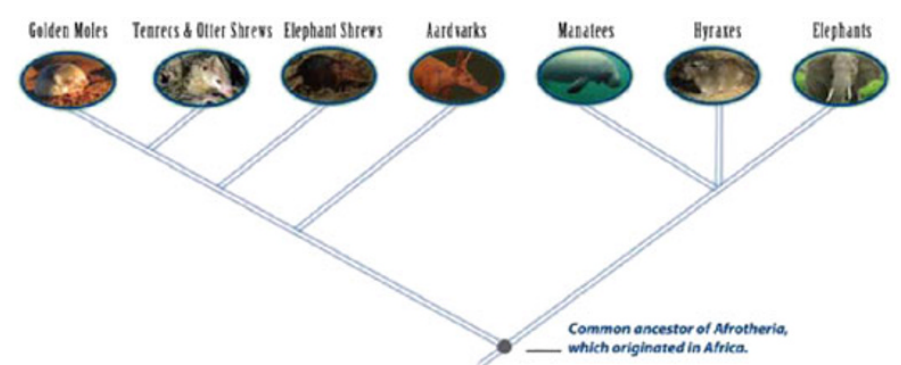

(a) Cladogram (Used with permission of Yale Peabody Museum of Natural History)

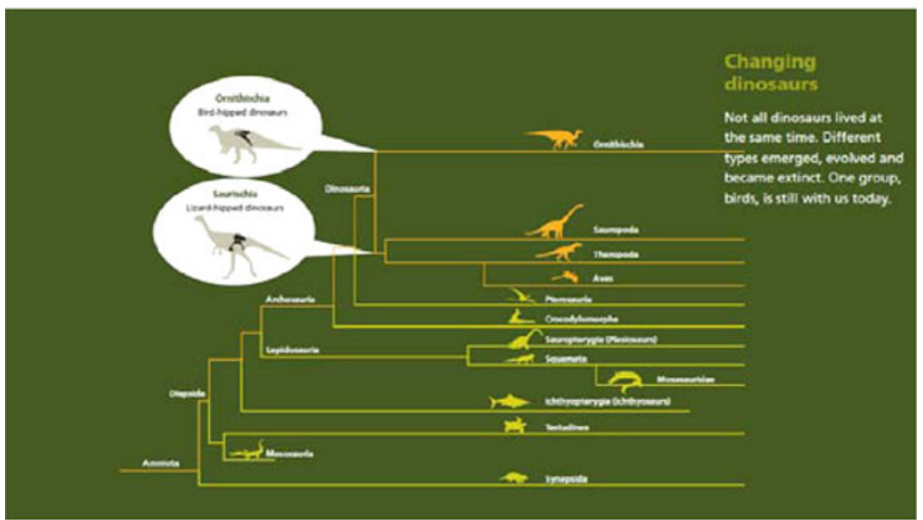

(b) Almost-a-cladogram

(Courtesy Australian Museum, different terminal end points)

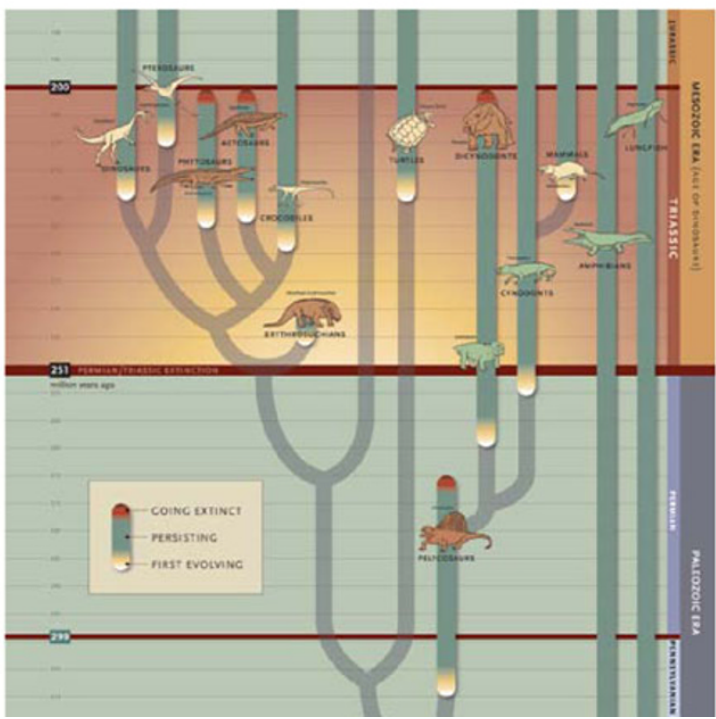

(c) Non-cladistic/other evolutionary tree

(Courtesy New Mexico Museum of Natural History \& Science, multiple taxa along branch) institution and with considerable variation in content, annotation, and presentation. This use of varied tree forms is also found in formal education contexts in which different depictions of the tree of life-in some cases inaccurate and misleading ones-are presented in textbooks, often alongside variable biological classification systems (Catley and Novick 2008).
Tree Type

Overall, most museum trees are represented as cladograms in the broadest sense $(61.6 \%, n=114$ of 185$)$-cladogram and almost-a-cladogram categories together-much less frequently compared to the $72 \%$ in biology textbooks (Catley and Novick 2008). However, fewer than half are considered to be strict 
Table 1 Categories, criteria, and coding used for museum trees

\begin{tabular}{ll}
\hline Category & Description and coding \\
\hline $\begin{array}{l}\text { Topological and diagrammatic elements } \\
\text { Orientation }\end{array}$ & \\
& $\begin{array}{l}\text { Overall orientation of tree, or the position of the root } \\
\text { relative to branches. Those with no overall orientation } \\
\text { (e.g., circular or radial geometries) were coded as N/A }\end{array}$ \\
Overall direction of branches from the root; circular trees \\
were coded by the direction of the initial spiral, and radial \\
trees were coded N/A \\
Trees classified as a cladogram and "almost-a-cladogram" \\
were coded as angled, rectangular, curvogram/swoopogram, \\
circular, radial, or eurogram. Non-cladistic/other evolutionary \\
trees, were coded as N/A \\
Whether branches end at different levels \\
Taxa are represented visually (graphically through images, \\
silhouettes, or with models/specimens) \\
Diagrams has a central main trunk with taxa branching \\
off of it with a clear linear progression from "lower" to \\
"higher" forms (Haeckel 1874)
\end{tabular}

\section{Tree content}

Anagenesis

Taxa

Extinct taxa

Humans and their most recent extinct relatives Geological time

Classification

Common ancestor

Synapomorphies

Hybridization

\section{Presentation and explanation \\ Exhibit component}

Instructional information/interpretation

Nature of science
Depicts ancestor-descendant relationships between named taxa (e.g., genus or species) with one or more named taxa in a sequence along a branch

Invertebrates, vertebrates, broad taxonomic categories, or other (e.g., viruses)

Includes extinct taxa

Includes one or more members of this group

Includes an indication of time

Explicit links between parts of tree and more familiar classifications of organisms

Refers to one or more common ancestors

Synapomorphies (shared characteristics) are indicated

Includes lateral transfers of genetic material, i.e., it represents a phylogenetic network in which hybridization or similar events are believed to have been involved, rather than a tree that only depicts branching sequence

Static flat graphic panel, graphic backdrop for specimens/ models, 3D representation, media component (e.g., video or game in kiosk/online), or a supplemental document

Provides an explanation of what the tree shows (e.g., refers to relationships between taxa, describes changes or trends over time), instructs how to interpret evolutionary diagrams

(e.g., describes trees as branching diagrams that show relatedness)

Labels or legends include information about the data used to build the tree, refers trees as hypotheses or product of scientific reasoning cladograms $(26 \%, n=29)$. Catley and Novick (2008) expressed concern over the use of "almost-a" cladogram format due to its potential to create confusion about cladistic principles and misinterpretation of diagrammatic elements such as varying branch length. The occurrence of other forms of evolutionary tree diagrams (non-cladistic) in museums $(38.4 \%, n=71)$ is correspondingly higher than the $28 \%$ found in textbooks. Many of the diagrams in this category are challenging to decipher, with some diagrammatic elements not labeled or subject to alternative interpretations, which makes it difficult 


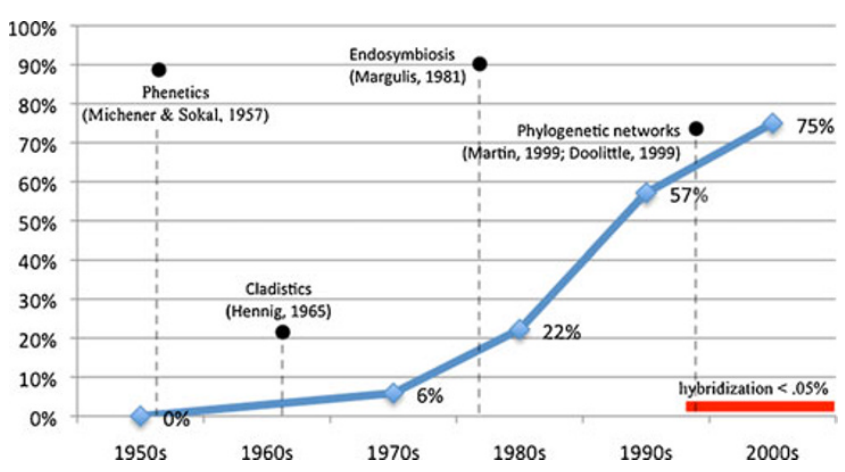

Fig. 3 Percentage of museum trees that are cladograms (sensu lato) over time

to determine the designers' intentions or consider what a visitor might take away from their experience with it.

Figure 3 shows the frequency of cladograms over time as a percentage of the sample trees collected, with the publication of significant systematic papers indicated. There are a few trends to note: cladograms (sensu lato) appear in museums in the 1970s, but not with any frequency until the 1990s; hybridization is rare in museum trees, but appears at the same time as publications about phylogenetic networks; and while the use of non-cladistic diagrams has declined, they remain a significant part of more recent exhibits, representing almost a third of graphics within the last decade.

\section{Tree Orientation and Direction}

Most exhibit trees have a clearly discernable orientation and direction from root to tip; the majority being oriented both vertically and upward $(n=124)$. Of the 49 horizontal trees, most are organized in a left-to-right direction $(n=46)$.

\section{Tree Geometry}

Different geometries can be used show identical relationships, and multiple names may refer to the same format depending on the particular software program and researcher preference (see Table 2). Trees of the cladogram and almost-a-cladogram ( $n=114$ of 185$)$ categories could be coded by geometry. Most use either a rectangular (45.6\%, $n=52)$ or angled format $(37.7 \%, n=43)$-referred to as tree and ladder, respectively, by Catley and Novick. This differs somewhat from cladograms in biology textbooks (Catley

Table 2 Phylogenetic tree geometry (descriptions modified from tree software sites, e.g., Phylodendron, Drawgram, etc.)

\begin{tabular}{|c|c|c|}
\hline Example & Description & Names used \& sources \\
\hline & $\begin{array}{l}\text { Nodes connected to other nodes and to tips by } \\
\text { straight lines directly from one to the other. This } \\
\text { category includes diagrams with slightly wavy } \\
\text { lines or curved lines, but have an overall pectinate } \\
\text { layout. }\end{array}$ & $\begin{array}{l}\text { - Angled (e.g., PhyloDraw, TreeView) } \\
\text { - Slanted (e.g., PhyloDraw, TreeView) } \\
\text { - Cladogram (e.g., Drawgram, Phylodendron) } \\
\text { - Diagonal (e.g., Mesquite) } \\
\text { - Ladder (Catley and Novick 2008) }\end{array}$ \\
\hline & $\begin{array}{l}\text { Nodes connected to other nodes and other tips by a } \\
\text { horizontal and then vertical line. This category } \\
\text { includes diagrams with slightly curved corners } \\
\text { and/or wavy branches. }\end{array}$ & $\begin{array}{l}\text { - Rectangular (e.g., PhyloDraw, TreeView) } \\
\text { - Square (e.g., Drawgram, Mesquite) } \\
\text { - Phenogram (e.g., Drawgram, Phylodendron) } \\
\text { - Tree (Catley and Novick 2008) }\end{array}$ \\
\hline & $\begin{array}{l}\text { Nodes connected by curves that are } 1 / 4 \text { of an } \\
\text { ellipse; curvogram starts horizontally then curves } \\
\text { up to become vertical; first } 1 / 3 \text { of swoopogram } \\
\text { starts out horizontal then vertical then follows } \\
\text { curvogram. }\end{array}$ & $\begin{array}{l}\text { - Curvogram/Swoopogram (e.g., Drawgram, } \\
\text { Phylodendron) } \\
\text { - Angular curvograms/Curved curvograms (e.g., } \\
\text { TreeDom) }\end{array}$ \\
\hline & $\begin{array}{l}\text { Nodes connected outwards from a central point, } \\
\text { with tips forming a circle. Radial lines run outward } \\
\text { from the center with the arc segments centered on } \\
\text { them. }\end{array}$ & - Circular (e.g., Phylodraw, TreeView, PAUP) \\
\hline & $\begin{array}{l}\text { Nodes connected outwards from a central point } \\
\text { without horizontal lines. }\end{array}$ & - Radial (e.g., Phylodraw, TreeView) \\
\hline & $\begin{array}{l}\text { Nodes connected to other nodes and to tips by a } \\
\text { diagonal line that goes outwards to at most } 1 / 3 \text { of } \\
\text { the way up to the next node, then turns sharply } \\
\text { straight upwards and is vertical. }\end{array}$ & - Eurogram (e.g., Drawgram, Phylodendron) \\
\hline
\end{tabular}


and Novick 2008), which found a consistent preference for angled over rectangular diagrams (55\%) across grade levels. However, if we only consider strict cladograms, then angled and rectangular trees in museums are equal ( $n=18$ for each), 11 are eurograms, and two are circular. Compared to textbooks, museums seem to use a wider variation of cladogram geometries including curvogram/swoopogram and eurogram.

Trees and Time

Fewer than half of the trees ( $n=85$ of 183) include time as a timeline on the diagram (see Fig. 2c), as labels along branches or at nodes, or in association with information about taxa in the tree (e.g., specimen labels), with another $20 \%(n=37)$ referring to time in associated label text. This is consistent with the $42 \%$ of biology textbook diagrams found to include some representation of time (Catley and Novick 2008).

In addition to the explicit labeling of a time axis or as data points on a diagram, absolute time may be implied by variation in branch length between extinct and extant taxa (see Fig. 2b). Variation in branch length (differing end points for terminal taxa) and the inclusion of time on the tree diagram were significantly associated in the sample (Fisher's test, $d f 1, n=185, p<0.001$ ). Furthermore, the inclusion of extinct taxa is significantly correlated with variation in branch length (Fisher's test, $d f 1, p<0.001$ ), suggesting that differing branch length is being used as a diagrammatic representation of an absolute or relative time dimension; however, in many cases, extinct taxa are not labeled as such. Trees with only vertebrates are significantly more likely to include extinct taxa (Fisher's test, $d f 1, n=$ $184, p<0.001$ ), and so there may be an expectation that museum visitors are more familiar with extinct vertebrates (e.g., dinosaurs, mammoths) than with other organisms.

\section{Tree Content and Labeling}

Many museum trees include additional information beyond showing common ancestry and relatedness between taxa. Examples include labeling nodes with specific or representative hypothetical common ancestors (see Fig. 2a); highlighting the synapomorphies (shared derived characters, see Fig. 2b) that support the proposed relationships, suggested hybridization paths or events; as well as the diversity, geographical distribution, and diet of different groups. Of the 185 trees in the sample, three are not yet finalized and so were excluded from the analysis of some content categories.

Close to $40 \%$ of the trees refer to ancestors/common ancestors $(n=72$ of 182$)-14.6 \%(n=27)$ on the diagram itself and $24.3 \%(n=45)$ in associated text. Only $20 \%$ of museum trees label synapomorphies (shared characters) that support the relationships on the tree $(n=37$ of 182), and another $23 \%(n=43)$ refer to particular shared characters in the text. Links to classification were found in over half of museum trees collected (55\%, $n=102$ of 182). In terms of taxonomic groups represented, most trees include only vertebrates $(73 \%, n=135$ of 185$)$, followed by the overall relationships between broad categories across the taxonomic spectrum $(15.7 \%, n=29)$, and then invertebrate animals $(7.6 \%, n=14)$; only a small number of trees $(3.8 \%, n=7)$ show other groups of organisms such as viruses.

Hybridization - exchange between lineages such as gene transfer and hybridization between species - is absent from most museum trees (95\%, $n=176$ of 182). The absence of hybridization is not surprising given that most trees of life do not reflect this complexity of evolution (Brooks and Hoberg 2008; Grant and Grant 2002). Furthermore, most museum trees focus on vertebrates for which the general consensus is that hybridization plays only a minor role (Dowling and Secor 1997). The six museum diagrams that do show hybridization are from the late 1990s and 2000s, three of which specifically refer to hybridization in the diagram or in associated explanatory text (see Fig. 4).

\section{Tree Presentation}

Of the 185 evolutionary the trees collected, $89.2 \%(n=165)$ are part of onsite exhibitions, most of which take the form of tree diagrams on flat graphic panels $(73.5 \%, n=136)$ with $15.1 \%(n=28)$ incorporating specimens or models into the tree; only two are represented as three-dimensional structures - one as a single exhibit piece (Fig. 5a), the other as a series of connected branches throughout the exhibit (Fig. 5b). More than $80 \%(n=151)$ incorporate visual representations of the taxa in the form of specimens, models, illustrations, or photographs. Fourteen trees $(7.6 \%)$ were media based as videos or games accessible either online, via an onsite kiosk (Fig. 5c) or occasionally both. Typically, the user can step through presented information or navigate different parts of the tree.

\section{Tree Explanatory Information}

Close to $70 \%$ of the trees $(n=125$ of 182$)$ include some kind of description or explanation about what the tree shows, or refer to trees as branching diagrams that show relationships; however, for many, the link between the tree and the exhibit of which it is part of is unclear. Of those that do provide some explanation, just over $50 \%(n=67)$ make explicit reference to the particular tree shown. Over two thirds of exhibits $(n=121$ of 182$)$ do not make any reference to the tree being a result of scientific research or that it represents a hypothesis. 


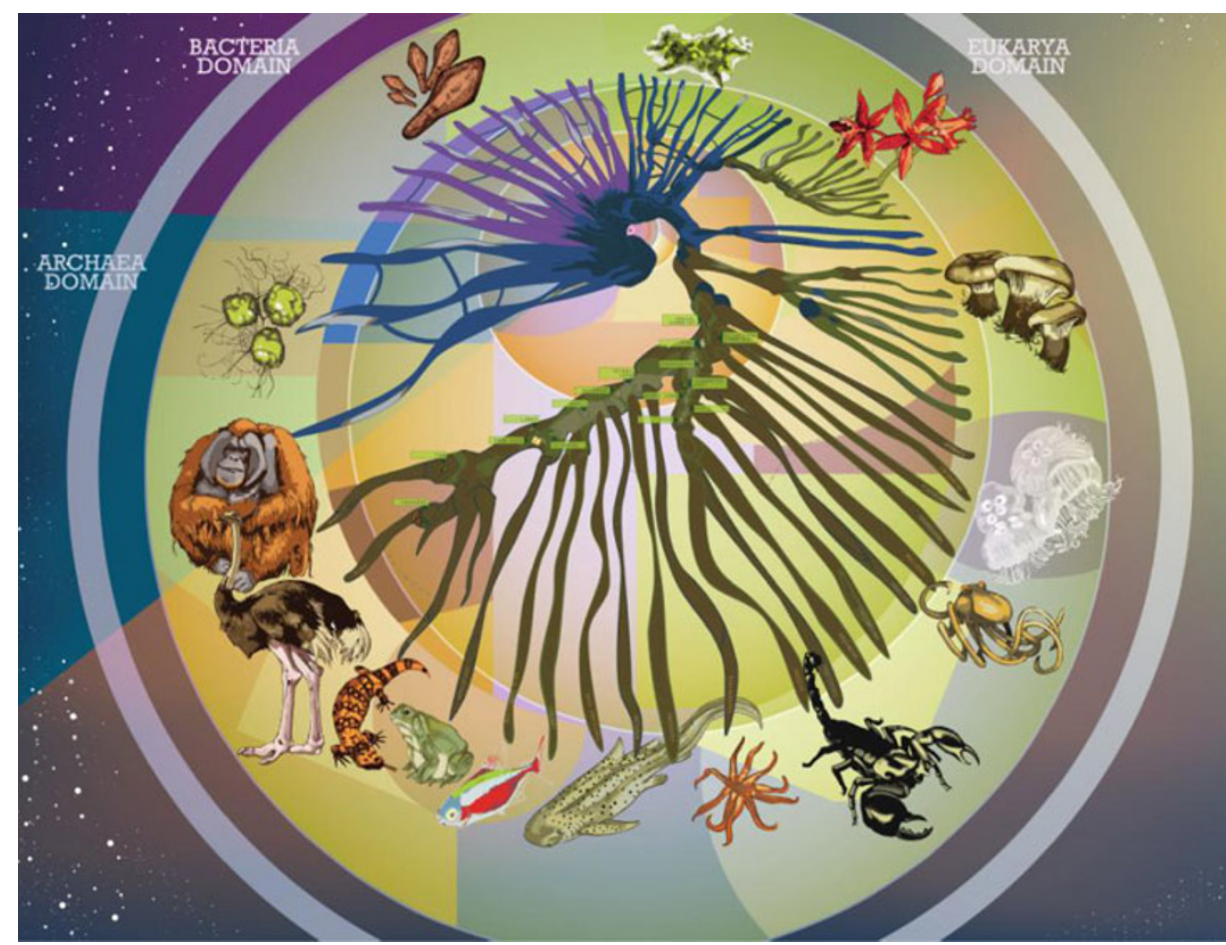

Courtesy of Dusquene University (Pittsburgh Zoo and PPG Aquariums), all life (2009). Credits: Art director/lead art—Joana Ricou; executive director-John Pollock; research—Brinley Kantorski, Allison Pogue; additional art-Robert Hoggard.

Fig. 4 Example of a tree graphic that depicts hybridization

\section{Discussion}

\section{Monophyletic Groups}

In the most basic sense, evolutionary trees are branching diagrams showing common ancestry and the relationships between taxa-with variations on this theme depending on the scientific statements being proposed and have a variety of terminology associated with them (e.g., cladograms, phylograms, etc.). One central idea to consider when trying to think about trees phylogenetically, from an evolutionary science and educational perspective, is monophyly (see Wiley 1979, 1981, 2010).

The concept of monophyly as an organizational framework for studying relatedness forms the foundation of phylogenetic thinking, but is often not reflected in classification systems. Donovan and Wilcox (2004) suggest that links to classification in tree diagrams may support the recognition of biological patterns, and research suggests that teaching classification independent of phylogeny supports the development and persistence of alternative conceptions about animal classification (Brumby 1984; Griffiths and Grant 1985; O'Hara 1992; Trowbridge and Mintzes 1988; Wellman and Gelman 1998; Wiley et al. 1991; Yen et al. 2004). The classification schemes that adults and children are exposed to, and most familiar with — such as that birds belong to their own class, Aves, separate from Reptilia — do not reflect the principle of monophyly. The absence of monophyletic groups as an organizational framework for organisms is thought to be particularly problematic for developing an understanding of evolution (American Association for the Advancement of Science 2001; Catley et al. 2005).

More than half of museum trees make links between tree sections and traditional classification categories, and many textbooks present trees alongside widely varying biological classification systems (Catley and Novick 2008). Furthermore, Sandvik (2007) argued that textbooks often adjust the resolution of cladograms - collapse different parts of the tree - to reflect more familiar Linnaean categories, and so these taxa are overrepresented in the diagrams. Whether the predominance of vertebrates in museum trees reflects a deliberate pruning to focus on more familiar Linnaean groups, popular taxa, or institutional research focus is unknown.

From a genealogical perspective, a meaningful classification would reflect monophyletic groups, and the idea of similarity should be understood through the principle of phylogeny. The mismatch between classification and phylogeny can result in grouping by arbitrary (or at least not in evolutionarily meaningful) ways and leads to confusion 
Fig. 5 Examples of tree

presentation formats: a $3 \mathrm{D}$ tree,

b 3D tree, and c media kiosk

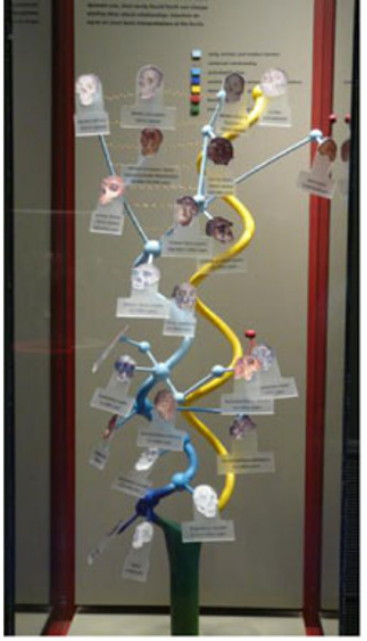

(a) Courtesy of the Frank H. McClung Museum, The University of Tennessee, Knoxville(2004)

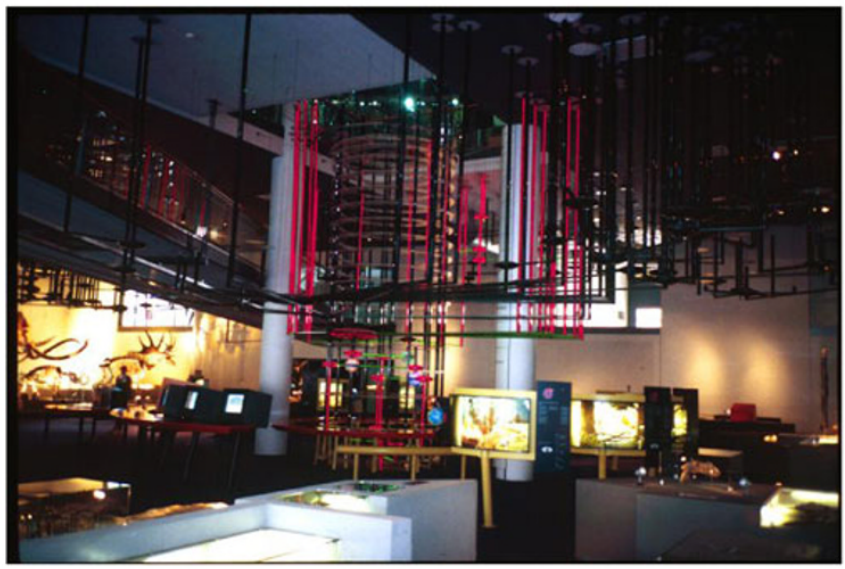

(b) Courtesy of Naturalis (1998)

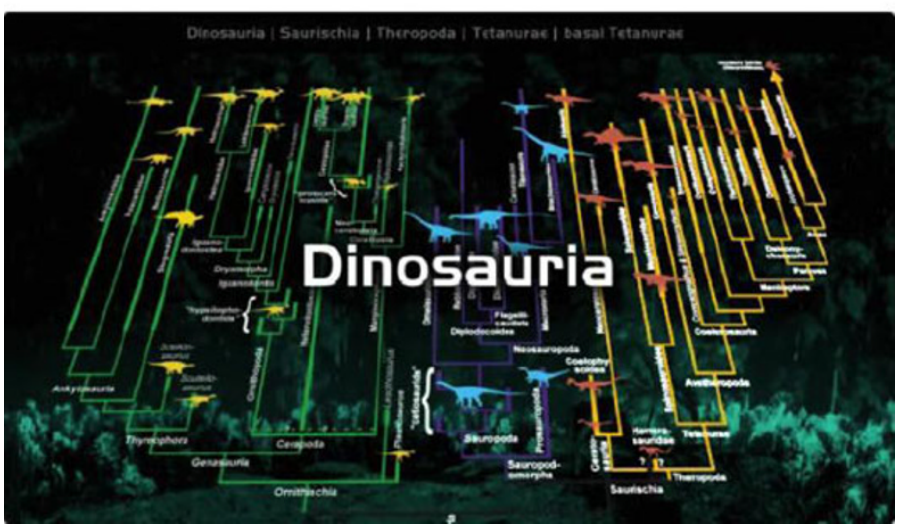

(c) Carnegie Museum of Natural History (2008). (C) Carnegie Museum of Natural History. about shared derived features and convergent similarities. Presenting a phylogenetic tree in conjunction with classification may help novices make connections between the tree and more familiar ideas and ways of thinking, but how best to convey this when these classifications conflict with the statements of relationships depicted in trees is a challenge.
Tree Iconography

Images can be powerful tools for communicating ideas, but their interpretation and understanding are influenced by context and prior conceptions. Visitors' experiences and understanding of exhibits are framed within a wider cultural 
framework-with museums challenging or supporting existing knowledge. For example, a study of human evolution museum exhibits by Scott $(2007,2006)$ found that information about evolution is obtained from a wide range of sources including TV, films, books, family discussions, and museums, and these conceptions influenced visitors' interpretation and understanding of these exhibits.

Some authors suggest that many of the icons used in evolutionary diagrams - cones of increasing diversity (i.e., trees with narrow bases and wide tops), upwardly directed trees, and trees with differential resolution (emphasizing some taxonomic groups) - reinforce ideas of evolution as progressive and directional (Gould 1995, 1997; O'Hara 1992). Matuk (2007) and Clark (2001) in their discussions of evolutionary images note that the simplified representations of horse evolution that suggest a straightforward and linear progression, first presented in the early 1900s, persists today. Indeed, horse evolution diagrams that depict anagenesis, ancestor-descendant sequences, with taxa arranged sequentially along a time scale, continue to be used in textbooks (Catley and Novick 2008) and are found in museum exhibits.

Unlike biology textbooks (Catley and Novick 2008), "Tree of Life" depictions - diagrams with a central trunk and a distinct "progressive" branching sequence from "lower" organisms on the bottom to "higher" ones at the top (a.k. a "Great Chain of Being" or scala naturae) - were not found in this sample of museum trees. However, two exhibit diagrams have what might be interpreted as vertical, hierarchical representations of primates with a central core and side branches with prosimians at the bottom and apes at the top (Fig. 6). What significance, if any, visitors might attribute to these particular examples is unknown, but previous work has demonstrated the potential for interpreting the layout of exhibits that include humans, their most recent extinct relatives, and/or other primates as directional and progressive (Scott and Giusti 2006).

\section{Orientation and Direction}

While identical evolutionary relationships can be depicted using any tree orientation and direction and/or geometrical shape, the particular form used may have implications for its accessibility to users and impact the interpretation of information shown in the diagram. Spatial framework theory suggests that the directions used to refer to something are based on the participants using their body as a reference point and that biases in our perceptions of horizontal and vertical space result from our conceptual representations of those spaces (Franklin and Tversky 1990; Tversky 2002, 2005a). Cross-cultural studies have found that directionality varies by concept and language but that both children and adults map temporal increases horizontally on diagrams, with the direction of time reflecting the direction of their

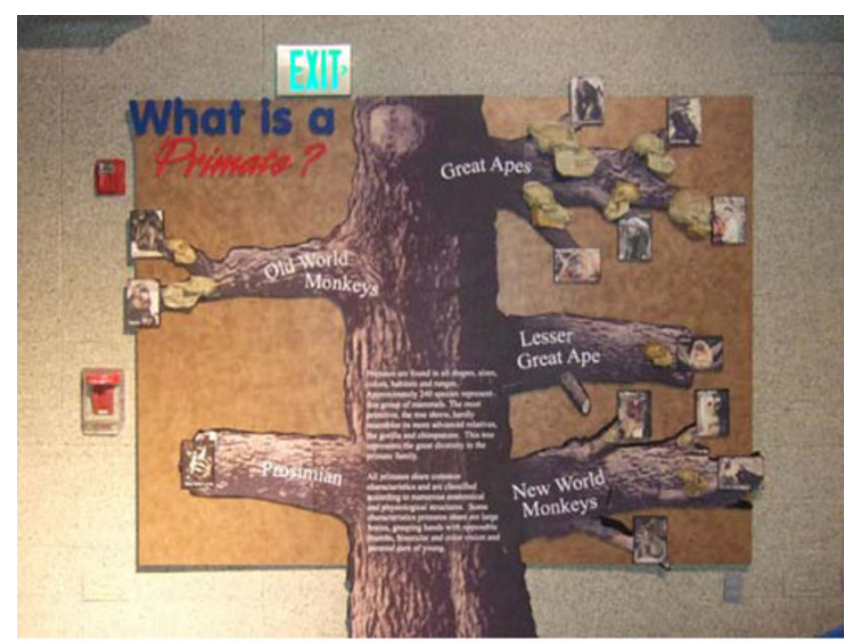

Courtesy Omaha’s Henry Doorly Zoo (2004)

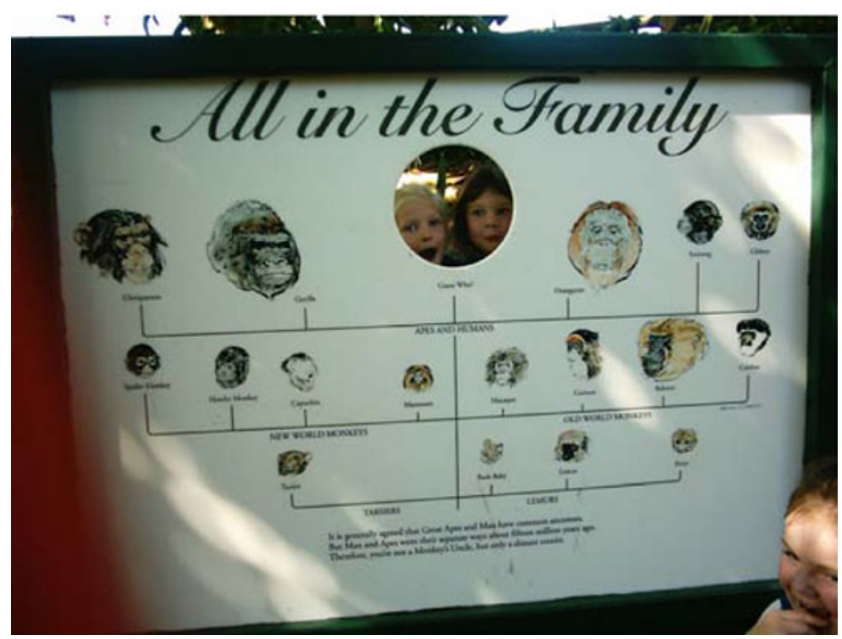

Courtesy Santa Barbara Zoo (1996)

Fig. 6 Examples of primate trees with a central trunk and side branches

written language (Tversky 2001, 2005b; Tversky et al. 1991).

The potential implication for orientation of tree diagrams is two-fold: misreading of time direction and the potential for reinforcing linear and progressive conceptions of evolution. The misreading of time across the top from left to right (in vertically oriented trees) rather than from bottom-up is a common misconception in interpreting tree diagrams (Giusti and Scott 2006; Gregory 2008; Meir et al. 2007).

The majority of museum trees sampled were oriented vertically with branches directed upward from the root. It is possible, given perceptual biases of horizontal and vertical space, that the tendency toward using vertical and upwardly directed diagrams contributes to this common error in reading temporal direction on trees. Vertically oriented diagrams have the potential to create confusion about the direction of time, particularly when not all trees explicitly label time, either absolutely or relatively. Many tree of life 
depictions in biology textbooks have no direct indicator of time, leaving it to the user to determine the relative time direction which may be incorrectly inferred (Catley and Novick 2008). In this study, fewer than half of museum trees label time on the diagram, but many depict time diagrammatically through variation in branch length for extinct and extant taxa.

Also, it is possible that vertical trees have the potential to reinforce ideas of progression and direction in evolution, as vertically oriented diagrams are often associated with quantitative increases and notably correspond to the linguistic metaphors of "up" and their associations with concepts of "more" and "better" (Tversky et al. 1991). The idea that evolution is a directional process from lower/primitive to higher/advanced is a powerful cultural narrative, often mirrored in popular imagery about evolution (Clark 2001; Gould 1997; Green and Shapely 2005; Matuk 2007; O’Hara 1992).

However, Phillips et al. (2010) found that the layout of terminal taxa in a cladogram that is oriented horizontally from root to terminal points - so that the taxa are organized vertically along the edge - elicits more frequent teleological responses and explanations from students than cladograms oriented vertically from the root, where terminal taxa are organized on the horizontal. Therefore, the authors suggest using cladograms with terminal taxa oriented horizontallya vertical root to branch orientation - and the placement of more complex taxa in the middle to help avoid teleological thinking. These results support the embodied cognition perspective discussed earlier (Franklin and Tversky 1990) but differ in the tree element being considered in the context of orientation - overall tree or resultant layout of terminal taxa.

Furthermore, learning research has found that reasoning about evolution differs by organism (Diamond and Evans 2007) and that the interpretation of cladograms is impacted by users' prior knowledge, and their narratives about evolution are typically overlain onto tree diagrams (Matuk 2008a, b, c; Matuk and Uttal 2012). The relative importance of overall tree orientation and conceptions of diagrammatic space and the layout of terminal taxa as a result of that orientation - and how either or both may be amelioratedwarrants further consideration.

In addition to orientation, geometry has implications for tree understanding. While different geometries show equivalent relationships and the selection of one versus another may be arbitrary, the particular form used may have implications for interpretation. Novick and Catley (2007) found that undergraduate students had greater difficulties extracting the hierarchical structure and relationships in angled cladograms than rectangular ones (what they refer to as ladders and trees, respectively) despite their being equivalent in terms of the information they contain. The authors suggest that the difficulty in seeing the nested relationships in the ladder results from the Gestalt principle of good continuation. Good continuation implies that the sloped line at the base of the ladder/angled diagram represents a single hierarchical level rather than the multiple levels it actually represents. The principle of good continuation then acts as a cognitive constraint resulting in the straight line being seen as a unit that continues without change, making it difficult for students to understand and interpret the relationships being depicted. Angled cladograms were also found to be more likely to elicit anagenic responses - speciation by transformation of one form into another-than rectangular ones (Novick et al. 2010b).

\section{Humans in Evolutionary Trees}

Museum visitors' reasoning about organisms and evolutionary explanations varies depending on the taxa included in the tree diagram, particularly humans (Diamond and Evans 2007). How visitors perceive exhibits with humans and other living or extinct primates in them is complex and challenging, but they are often interpreted as being linear, directional, and progressive (Scott 2007, 2010; Scott and Giusti 2006).

In addition to the common vertical orientation of trees, the location of Homo sapiens and other hominin species in relation to the other taxa in the tree has the potential to reflect and reinforce ideas of teleology and progression (Matuk 2007; Tversky 1995). A survey of textbook charts found most to be vertically organized with $H$. sapiens at the top (Tversky 1995), and an analysis of anthropocentrism in phylogenetic textbooks found the position of humans on the top-right of the left-right axis of vertical cladograms to be significant (Sandvik 2007). In museum trees, a bias for topright placement of humans was not found; however, the sample size was small $(n=9)$.

The common misreading of time across the top of a cladogram from left to right-coupled with reading the order of terminal taxa across the top as relatedness - may be interpreted as a progression from "old, primitive or simple" to "recent and complex," culminating in humans (Baum et al. 2005; Catley and Novick 2006; Giusti and Scott 2006; Halverson et al. 2008; Meir et al. 2007). Furthermore, a recent study of the impact of taxa placement in cladograms found that students were more likely to provide teleological responses and explanations if humans occupied an end, rather than a central location (Phillips et al. 2010).

In addition to the placement of $H$. sapiens, the portrayal of hominin evolution as primarily anagenic, by depicting one or more taxa placed on or within a single branch, is problematic for its potential to reinforce ideas of teleology, progression, and anthropocentrism. While anagenesis is common in textbook trees with humans (Catley and Novick 2008), fewer than a third of museum trees that include humans depict anagenesis. However, of those that do, all 
include $H$. sapiens and their most recent extinct relatives (e. g., Homo, Australopithecus, etc.) rather than humans in relation to other extant primates or other taxa.

\section{Geological Time}

Time is an important and difficult concept in understanding evolutionary trees, and the interpretation of time on trees is influenced by a range of factors including branch length and naïve understanding of evolutionary processes (Dodick 2010). It has been suggested that where temporal data are available, the inclusion of geological time on diagrams may help to support understanding (Catley and Novick 2008) and help with the common misreading of time across the top rather than bottom-up in vertically oriented trees (Meir et al. 2007).

Variation in branch length is thought to have the potential to promote understanding if the earlier ending points indicate extinct taxa (Catley and Novick 2008), and the inclusion of extinct taxa could help to avoid ideas of species persistence and progress (Donovan and Hornack 2004) - in part because a long branch is often incorrectly interpreted as a lineage in which no change has occurred (Crisp and Cook 2005; Novick and Catley 2007). However, the potential value of different branch length to identify extinct groups may be hampered by the fact that the significance of this diagrammatic feature is often not made explicit. Recent research indicates that there is a strong correlation between understanding the direction of time and the ability to explain evolutionary problems as represented in phylogenetic diagrams and that explicitly including temporal information on diagrams may support understanding and avoid the common misreading of relatedness along the tips, a.k.a tip-reading (Dodick 2010). The interpretation of time in phylogenetic trees and advantages and disadvantages of explicitly doing so are subject to much discussion, and continuing research will help to clarify these issues (Catley and Novick 2008; Dodick 2010).

\section{Tree Content and Labeling}

Most of the museum trees do not label tree components such as the root or node(s) as representing common ancestors or shared derived characters (synapomorphies) between taxa. Fewer than half refer to ancestors/common ancestors-with less than $15 \%$ included on the diagram itself-and only $20 \%$ labeling specific synapomorphies that support the relationships depicted on the tree. Donovan and Wilcox (2004) suggest that labeling the root or other internal node as "common ancestor" can help to overcome the abstractness of tree representations and support the interpretation of nodes. Others argue that since the ancestor is unknown, it is disingenuous to include it (Catley and Novick 2008), and doing so has the potential to reinforce the view of nodes as precise moments of change (Meir et al. 2007). Recent research has found that the inclusion of synapomorphies can help support understanding of tree diagrams and that evolutionary relationships are based on shared characteristics (Novick et al. 2010a), making their relatively uncommon use in museum tree diagrams problematic in terms of supporting visitor understanding.

Museum trees often include, in graphic form, other information beyond relatedness and common ancestry, such as diversity, by altering variables such as branch length, thickness, or shape and using color-coding and symbols. These are often not made explicit on the tree itself or in an associated legend or key. Textbook trees often use branch thickness to indicate diversity, but the graphical significance of this is generally unclear and undefined (Catley and Novick 2008). The absence of clear labeling means that the significance of these variables, if any, may be unclear, which makes it difficult to read and interpret the diagram. Being explicit about the intent of abstract diagrammatic elements is likely to aid in tree interpretation.

\section{Tree Explanatory Information}

For most museum trees, the exhibit text describes what can be seen in the tree-e.g., which taxa are most closely related -but the link to the graphic itself is usually not explicit. Evaluation studies suggest that it is important to directly tie labels to what visitors can experience at that point in the exhibition (McLean 1993; Serrell 1996), and presenting explicit information and concrete ideas in exhibit labels helps to instruct visitors about what they should look for (Bitgood 2000; Falk 1997; Falk and Dierking 1992). However, the lack of explicit annotation in many museum trees is not surprising given its absence in most evolutionary diagrams used in textbooks (Catley and Novick 2008), although its inclusion could support an understanding and interpretation of evolutionary processes (Donovan and Hornack 2004). Overall, the absence of explicit explanations for many trees or information about trees as products of science is likely to add to the difficulty that visitors have in reading and understanding of these diagrams.

\section{Tree Presentation}

Overwhelmingly, tree diagrams used in museum exhibits are part of graphic panels with images or specimens/models of taxa at terminal taxa points. Incorporating visuals into trees may draw attention to the organisms, help users to recognize and identify taxa, and assist visitors in connecting labeled synapomorphies with visible morphological characteristics. Many novices emphasize morphological features and similarity-based reasoning in their thinking about biological 
relationships, and so caution should be used to avoid conflating overall similarly with relatedness (Gelman 2004; Gelman and Markman 1987; Halverson et al. 2008; Sloutsky et al. 2001); however, explicitly labeling synapomorphies that are used to support the relationships shown in the tree-and perhaps that can be seen in accompanying visuals - may help highlight the evidence used in tree building, that of relatedness based on shared derived characters, and support ideas about scientific inference (Donovan and Wilcox 2004).

Fewer than $10 \%$ are multimedia-based, but some of these kiosks and online trees were interactive, where the user could step through the information or navigate to different parts of the tree. Summative evaluation of Yale's Travels in the Great Tree of Life exhibit found that the computer game exploring relationships was effective at communicating the idea that phylogenetic relationships may not always be what you might expect (Giusti 2008), which suggests that interactivity and/or animation may help address some issues with reasoning using trees. Based on personal experience with museum visitors, exploring the tree of life using manipulatives such as using scale models of taxa and different graphic representations can be effective with museum visitors. Research on the potential role of animation in understanding cladograms has found that animations can influence the perception and interpretation of diagrams, but that interpretation is also impacted by a user's prior knowledge and common evolution narratives (Matuk 2008a, b, c, 2010; Matuk and Uttal 2012).

\section{Conclusions and Further Work}

Museums seek to share current scientific research with the public and to teach visitors about evolution through their exhibits and programs. As this study and review of the literature shows, museums have a long history of using evolutionary graphics to communicate about relationships, and informal science institutions of all types are making efforts to incorporate evolutionary history or relatedness. Visitors are likely to be exposed to a variety of tree of life diagrams during a single museum experience. This diversity and the long standing use of trees of life in museums makes them an ideal setting to explore visitor understanding of these diagrams and to investigate strategies that can increase their effectiveness as tools for communicating about evolution and the tree of life.

Pilot studies from the Understanding the Tree of Life project provide some important insights into visitor understanding of trees, including the ability of young children to reason with tree diagrams, that trees can foster thinking about common ancestry and time- but may hinder an understanding of variation and selection, the impact of prior knowledge and existing narratives in interpreting trees, and the importance of time. These studies, and this review, highlight the importance and educational potential of evolutionary trees in museums and how much more work needs to be done. Further research is needed to explore how visitors interpret and understand these varied representations in a museum setting, and to understand what the visitors bring with them and how this can be used to support their understanding of phylogeny and the tree of life. However, the existing literature suggests three elements that might help to clarify visitor understanding of trees: (1) show time axis, (2) include shared characters, and (3) carefully consider the placement of taxa in trees, particularly humans.

The flexible and ubiquitous nature of informal learning provides a great opportunity to share current scientific knowledge and our understanding of the tree of life with the public - yet brings its own challenges, as these experiences occur within the context of visitors' prior knowledge and conceptions. As we strive to support the understanding of evolution with museum visitors, we need to think carefully about what we are trying to communicate, what role trees can play in supporting evolutionary thinking, and how this may be supplemented and supported by other exhibit components - in essence, how trees of life fit into the broader context of the visitor experience.

Acknowledgments Our thanks to Monique Scott (American Museum of Natural History) for inviting us to participate in this special issue on museums and evolution. Special thanks to Dawn Kirchner (University of Kansas) for her assistance and feedback through data collection and analysis and to Camillia Matuk (University of California-Berkeley), Sam Donovan (University of Pittsburgh), and Linda Trueb (University of Kansas) for their input. We would like to thank our reviewers for their helpful comments and suggestions. We also extend our sincere gratitude to all the institutions that shared their tree of life graphics, without which this research would not have been possible. This research was supported by the National Science Foundation-funded Understanding the Tree of Life project (grant no. 0715287). Any opinions, findings, and conclusions or recommendations expressed in this material are those of the author(s) and do not necessarily reflect the views of the National Science Foundation

\section{References}

American Association for the Advancement of Science. Atlas of science literacy, volume 1 (Vol. Project 2061). Washington, DC: American Association for the Advancement of Science (AAAS) and the National Science Teachers Association; 2001.

Baum DA, DeWitt-Smith S, Donovan S. The tree-thinking challenge. Science. 2005;310:979-80.

Bitgood S. The role of attention in designing effective interpretive labels. J Interpret Res. 2000;5(2):31-45.

Brooks DR, Hoberg EP. Darwin's necessary misfit and the sloshing bucket: the evolutionary biology of emerging infectious diseases. Evol Educ Outreach. 2008;1:2-9.

Brumby M. Misconceptions about the concept of natural selection by medical biology students. Sci Educ. 1984;68(4):493-503. 
Catley KM, Novick LR. Assessing undergraduate students' understanding of evolutionary concepts. Paper presented at the Hawaii International Conference on Education. 2006.

Catley KM, Novick LR. Seeing the wood for the trees: an analysis of evolutionary diagrams in biology textbooks. BioScience. 2008;58 (10):976-87.

Catley KM, Lehrer R, Reiser B. Tracing a prospective learning progression for developing understanding of evolution. Paper Commissioned by the National Academies Committee on test design for K-12 Science achievement. 2005. p. 67.

Clark CA. Evolution for John Doe: pictures, the public, and the Scopes trial debate. J Am Hist. 2001;87(4).

Crisp MD, Cook LG. Do early branching lineages signify ancestral traits? Trends Ecol Evol. 2005;20:122-8.

Diamond J, editor. The virus and the whale: exploring evolution in creatures small and large. Arlington: National Science Teachers Association (NSTA); 2005.

Diamond J, Evans EM. Museums teach evolution. Evolution. 2007;61 (6): $1500-6$

Diamond J, Scotchmoor J. Exhibiting evolution. Mus Soc Issues. 2006;1(1):21-48.

Diamond J, Evans EM, Spiegel A. Walking whales and singing flies: an evolution exhibit and assessment of its impact. In Rosengren KS, Brem S, Evans EM, Sinatra G, editors. Evolution challenges: integrating research and practice in teaching and learning about evolution. Oxford: Oxford University Press; in press.

Dodick J. Phylogeny exhibits and understanding geological time. Paper presented at the Understanding the Tree of Life conference. Pittsburgh: Carnegie Museum of Natural History; 2010.

Donovan S, Hornack D. Not losing the forest for the trees: learning to compare trees and assess support for phylogenetic hypotheses. Paper presented at the Society of the Study of Evolution meeting. Fort Collins; 2004.

Donovan S, and Wilcox L. Tree figures in texts: A framework for unpacking their educational potential. Poster presented at the Society for the Study of Evolution meeting. Fort Collins; 2004.

Dowling TE, Secor CL. The role of hybridization and introgression in the diversification of animals. Ann Rev Ecol Syst. 1997;28:593-619.

Falk JH. Testing a museum exhibition design assumption: effect of explicit labeling of exhibit clusters on visitor concept development. Sci Educ. 1997;81(6):679-87.

Falk JH, Dierking LD. The museum experience. Washington, DC: Whalesback; 1992.

Franklin N, Tversky B. Searching imagined environments. J Exp Psychol: Gen. 1990;119:63-76.

Gelman SA. Psychological essentialism in children. Trends Cogn Sci. 2004;8(9):404-9.

Gelman SA, Markman EM. Young children's inductions from natural kinds: the role of categories and appearances. Child Dev. 1987;58 (6): $1532-41$.

Giusti E. Travels in the great tree of life: a summative evaluation. New Haven: Yale Peabody Museum of Natural History; 2008.

Giusti E, Scott M. Tree of life visitor study. New Haven: Yale Peabody Museum of Natural History; 2006.

Gould SJ. Evolution by walking. Nat Hist. 1995;104(3):10-5.

Gould SJ. Redrafting the tree of life. Proc Am Philos Soc. 1997;141 (1):30-54.

Grant PR, Grant BR. Adaptive radiation of Darwin's finches. Am Sci. 2002;90:130-9.

Green D, Shapely RT. Teaching with a visual tree of life (final report). Berkeley: School of Information and Management Systems, University of California-Berkeley; 2005.

Gregory TR. Understanding evolutionary trees. Evol Educ Outreach. 2008;1:121-37.
Griffiths AK, Grant BAC. High school students' understanding of food webs: identification of a learning hierarchy and related misconceptions. J Res Sci Teach. 1985;22(5):421-36.

Haeckel EH. Anthropogenie. Leipzig, Germany: W. Engelmann; 1874.

Halverson KL. Using pipe cleaners to bring the tree of life to life. Am Biol Teach. 2010;72(4):223-4.

Halverson KL, Pires JC, Abell SK. Undergraduates' abilities to use representations in biology: interpreting phylogenetic tree thinking. Paper presented at the National Association for Research in Science Teaching. Baltimore; 2008.

MacDonald T. Communicating phylogeny: evolutionary tree diagrams in museums. Paper presented at the NARST (National Association for Research in Science Teaching) conference. Philadelphia; 2010.

Matuk CF. Images of evolution. J Biol Commun. 2007;33(3):E54-61.

Matuk CF. Animated cladograms: interpreting evolution from diagrams. Poster presented at the Fifth International Conference on the Theory and Application of Diagrams. Herrsching am Ammersee, Germany; 2008a.

Matuk CF. Animated cladograms: the perception and conception of evolution. Paper presented at the European Association for Learning on Research and Instruction (EARLI) conference. Tilburg, Netherlands; $2008 \mathrm{~b}$.

Matuk CF. Animating trees of life: how animation influences the perception of evolution. Poster presented at The International Conference on Spatial Cognition. Freiburgh, Germany; 2008c.

Matuk CF. Interpretation, invention, and interaction: using students (mis)understandings to redesign the tree of life. Paper presented at the Understanding the Tree of Life conference. Carnegie Museum of Natural History, Pittsburgh; 2010.

Matuk CF, Uttal DH. Manipulating narrative spaces in the representation and understanding of evolution (working title). In e. a. K. Rosengren (Ed.), Evolution challenges: integrating research and practice in teaching and learning about evolution. Oxford: Oxford University Press; in press.

McLean K. Planning for people in exhibitions. Washington, DC: Association of Science-Technology Centers; 1993.

Meir E, Perry J, Herron JC, Kingsolver J. College students' misconceptions about evolutionary trees. Am Biol Teach. 2007;69: $71-6$.

National Research Council. National education standards. Washington, DC: National Academy; 1996.

National Science Board. Science and engineering indicators. Arlington: National Science Foundation; 2008.

Novick LR, Catley KM. Understanding phylogenies in biology: the influence of a Gestalt perceptual principle. J Exp Psychol: Appl. 2007;13:197-223.

Novick LR, Catley KM, Funk DJ. Characters are key: the effect of synapomorphies on cladogram comprehension. Evo Edu Outreach; 2010a;3:539-47.

Novick LR, Shade CK, Catley KM. The effect of linear versus branching depictions of evolutionary history on students' interpretations of evolution as an anagenic process. Paper presented at the NARST (National Association for Research in Science Teaching) conference. Philadelphia; 2010b.

O'Hara RJ. Telling the tree: narrative representation and the study of evolutionary theory. Biol Philos. 1992;7:135-60.

Phillips BC, Novick LR, Catley KM, Funk DJ. Interactive effects of teleological beliefs and diagrammatic format on tree thinking. Paper presented at the Thirty-second Annual Meeting of the Cognitive Science Society. Portland, August; 2010.

Sandvik H. Anthropocentrism in cladograms. Biol Philos. 2007;24.

Scott M. Rethinking evolution in the museum: envisioning African origins. London: Routledge Taylor \& Franic Group; 2007.

Scott M. The pleasures and pitfalls of teaching human evolution. Evo Edu Outreach. 2010;3:403-9. 
Scott M, Giusti E. Designing human evolution exhibitions: insights from exhibitions and audiences. Mus Soc Issues. 2006;1(1):49-68.

Serrell B. Exhibit labels: an interpretive approach. Walnut Creek: Altimura; 1996.

Sloutsky VM, Lo Y-F, Fisher A. How much does a shared name make things similar? Linguistic labels and the development of inductive inference. Child Dev. 2001;72:1695-709.

Spiegel A, Evans EM, Gram W, Diamond J. Museum visitors' understanding of evolution. Mus Soc Issues. 2006;1:69-86.

Trowbridge JE, Mintzes JJ. Alternative conceptions in animal classification: a cross-age study. J Res Sci Teach. 1988;25(7):547-71.

Tversky B. Cognitive origins of graphic conventions. In: Marchese FT, editor. Understanding images. New York: Springer; 1995.

Tversky B. Spatial schemas in depictions. In: Gattis M, editor. Spatial schemas and abstract thought. Cambridge: MIT; 2001.

Tversky B. Some ways that graphics communicate. In: Allen N, editor. Words and images: working together differently. New York: JAI/ Ablex; 2002.

Tversky B. Form and function. In: Carlson LA, v.d. Zee E, editors. Functional features in language and space: Insights from perception, categorization and development. Oxford: Oxford University Press; 2005a. p. 400.
Tversky B. Functional significance of visuospatial representations. In: Shah P, editor. The Cambridge handbook of visuospatial thinking. Cambridge: Cambridge University Press; 2005b. p. 1-35.

Tversky B, Kugelmass S, Winter A. Cross-cultural and developmental trends in graphic productions. Cogn Psychol. 1991;23:515-57.

Wellman HM, Gelman SA. Knowledge acquisition and foundational domain. In: Kuhn D, Siegler R, editors. Handbook of child psychology. New York: Wiley; 1998. p. 523-73.

Wiley EO. Ancestors, species, and cladograms - remarks on the symposium. In: Cracraft J, Eldridge N, editors. Phylogenetic analysis and paleontology. New York: Columbia University Press; 1979. p. 211-26.

Wiley EO. Phylogenetics: the theory and practice of phylogenetic systematics. New York: Wiley; 1981.

Wiley EO. Why trees are important. Evo Edu Outreach 2010;3:499-505.

Wiley EO, Siegel-Causey D, Brooks DR, Fund VA. The Compleat cladist: a primer of phylogenetic procedures. Lawrence: The University of Kansas; 1991.

Yen CF, Yao TW, Chiu YC. Alternative conceptions in animal classification focusing on amphibians and reptiles: a cross-age study. Int J Sci Math Educ. 2004;2:159-74. 